\title{
Once more on the Witten index of $3 d$ supersymmetric YM-CS theory
}

\author{
A.V. Smilga ${ }^{1}$ \\ SUBATECH, Université de Nantes, \\ 4 rue Alfred Kastler, BP 20722, Nantes 44307, France \\ E-mail: smilga@subatech.in2p3.fr
}

ABSTRACT: The problem of counting the vacuum states in the supersymmetric $3 d$ YangMills-Chern-Simons theory is reconsidered. We resolve the controversy between its original calculation in [1] at large volumes $g^{2} L \gg 1$ and the calculation based on the evaluation of the effective Lagrangian in the small volume limit, $g^{2} L \ll 1[2]$. We show that the latter calculation suffers from uncertainties associated with the singularities in the moduli space of classical vacua where the Born-Oppenheimer approximation breaks down. We also show that these singularities can be accurately treated in the Hamiltonian Born-Oppenheimer method, where one has to match carefully the effective wave functions on the Abelian valley and the wave functions of reduced non-Abelian QM theory near the singularities. This gives the same result as original Witten's calculation.

Keywords: Supersymmetric gauge theory, Field Theories in Lower Dimensions, ChernSimons Theories

ARXIV EPRINT: 1202.6566

\footnotetext{
${ }^{1}$ On leave of absence from ITEP, Moscow, Russia.
} 


\section{Contents}

1 Introduction $\quad 1$

2 Effective action $\quad 5$

3 Effective Hamiltonian $\quad 8$

4 Torus with the corners $\quad 14$

$\begin{array}{ll}\text { A Theta functions } & 18\end{array}$

$\begin{array}{ll}\text { B Counting of states in pure CS theory } & 19\end{array}$

$\begin{array}{ll}\text { C Wave function at the origin } & 21\end{array}$

D Supercharges, Hamiltonian and gauge-invariant variables 22

\section{Introduction}

$3 d$ supersymmetric gauge theories attracted recently a considerable attention in view of newly discovered dualities between certain $\mathcal{N}=8$ and $\mathcal{N}=6$ versions of these theories and string theories on $A d S_{4} \times S^{7}$ or $A d S_{4} \times \mathbb{C P}^{3}$ backgrounds, respectively [3, 4].

In this paper, we discuss the simplest $\mathcal{N}=1$ version of such theories with nontrivial dynamics - the supersymmetric YM-CS theory with the Lagrangian

$$
\mathcal{L}=\frac{1}{g^{2}} \operatorname{Tr}\left\{-\frac{1}{2} F_{\mu \nu}^{2}+i \bar{\lambda} \not D \lambda\right\}+\kappa \operatorname{Tr}\left\{\epsilon^{\mu \nu \rho}\left(A_{\mu} \partial_{\nu} A_{\rho}-\frac{2 i}{3} A_{\mu} A_{\nu} A_{\rho}\right)-\bar{\lambda} \lambda\right\} .
$$

The conventions are: $\epsilon^{012}=1, D_{\mu} \mathcal{O}=\partial_{\mu} \mathcal{O}-i\left[A_{\mu}, \mathcal{O}\right]$ (such that $A_{\mu}$ is Hermitian); $\lambda_{\alpha}$ is a 2-component Majorana $3 d$ spinor belonging to the adjoint representation of the gauge group. We choose

$$
\gamma^{0}=\sigma^{2}, \quad \gamma^{1}=i \sigma^{1}, \quad \gamma^{2}=i \sigma^{3} .
$$

This is a $3 d$ theory and the gauge coupling constant $g^{2}$ carries the dimension of mass. The physical boson and fermion degrees of freedom in this theory are massive,

$$
m=\kappa g^{2} .
$$

In three dimensions, the nonzero mass brings about parity breaking. The requirement for $e^{i S}$ to be invariant under certain large gauge transformations (see e.g. ref. [5] for a nice review) leads to the quantization condition

$$
\kappa=\frac{k}{4 \pi} .
$$

with integer $k$. 
The first question to be asked for any supersymmetric theory is whether supersymmetry is spontaneously broken and, if not, what is the number of vacuum states. In most cases (and, in particular, in this case) the latter coincides with the Witten index

$$
I=\operatorname{Tr}\left\{(-1)^{F} e^{-\beta H}\right\}
$$

This index was evaluated in [1] with the result

$$
I(k, N)=[\operatorname{sgn}(k)]^{N-1}\left(\begin{array}{c}
|k|+N / 2-1 \\
N-1
\end{array}\right) .
$$

for $\mathrm{SU}(N)$ gauge group. This is valid for $|k| \geq N / 2$. For $|k|<N / 2$, the index vanishes and supersymmetry is broken. In the simplest $\mathrm{SU}(2)$ case, the index is just

$$
I(k, 2)=k .
$$

The result (1.6) was obtained by the following reasoning. Consider the theory in a large spatial volume, $g^{2} L \gg 1$. Consider then the functional integral for the index (1.5) and mentally perform a Gaussian integral over fermionic variables. This gives an effective bosonic action that involves the CS term, the Yang-Mills term and other higher-derivative gauge-invariant terms. After that, the coefficient of the CS term is renormalized, ${ }^{1}$

$$
k \rightarrow k-\frac{N}{2} .
$$

At large $\beta$, the integral is saturated by the vacuum states of the theory, which depend on the low-energy dynamics of the corresponding effective Hamiltonian. The latter is determined by the term with the lowest number of derivatives, i.e. the Chern-Simons term, the effects due to the YM term and still higher derivative terms being suppressed at small energies. Basically, the spectrum of vacuum states coincides with the full spectrum in the topological pure CS theory. The latter was determined some time ago

- by establishing a relationship between the pure $3 d$ CS theories and $2 d$ WZNW theories $[6]$

- by canonical quantization of the CS theory and direct determination of wave functions annihilated by the Gauss law constraints [7, 8]. To make the paper more self-sufficient, we briefly review the latter method in appendix B.

Then the index (1.6) is determined as the number of states in pure CS theory with the shift (1.8). For example, in the $\mathrm{SU}(2)$ case, the number of CS states is $k+1$, which gives (1.7) after the shift.

\footnotetext{
${ }^{1}$ This is for $k>0$. In the following, $k$ will be assumed to be positive by default though the results for negative $k$ will also be mentioned. The gauge coupling $g^{2}$ is also renormalized in some irrelevant way and new couplings (of still less relevant higher derivative terms) appear.
} 
In what follows, we will only consider the case $N=2$. A generalization of the analysis to other groups involves purely technical complications, which are, however, well understood and not controversial. We refer the reader to refs. $[2,7,8]$ for details.

Speaking of the controversy, it arises when the same problem is considered with a different method. Following the logics of [9], we considered the theory in a small volume, $\xi_{1,2} \in(0, L), g^{2} L \ll 1$, with periodic boundary conditions. The smallness of the parameter $g^{2} L$ allows one to apply the Born-Oppenheimer ideology and to evaluate the effective Lagrangian depending only on the relevant for low-energy dynamics slow variables. The slow bosonic variables represent in this case zero Fourier modes of spatial components of vector potential with zero classical energy. The latter implies that the field strength $\sim f^{a b c} A_{1}^{b(\mathbf{0})} A_{2}^{c(\mathbf{0})}$ is zero and $A_{j=1,2}^{a(\mathbf{0})}$ belong to the Cartan subalgebra. For $\mathrm{SU}(2)$, there is only one Abelian color component and there are only two bosonic slow variables $C_{j} \equiv$ $A_{j}^{\text {Cartan (0) }}$. All other modes are fast and can be integrated over.

Is it important that the slow configuration space is compact, the fields $C_{j}$ varying within the range $C_{j} \in(0,4 \pi / L)$. Indeed, a field outside this range can be brought into it by a large (i.e. not continuously deformable to unity, like $U(\boldsymbol{\xi})=\exp \left\{2 \pi i \frac{\xi_{1}}{L} \sigma_{3}\right\}$ ) gauge transformation. The effective theory describes then a motion ${ }^{2}$ over $T^{2}=S^{1} \times S^{1}$ with a, generally speaking, inhomogeneous magnetic field. This problem was analysed in $[10,11]$. For consistency (more exactly, for the spectrum to be supersymmetric [12]), the flux of the magnetic field should be quantized,

$$
\frac{\Phi}{2 \pi}=q=\text { integer }
$$

The Witten index of this Landau-Dubrovin-Krichever-Novikov theory coincides with $q$.

At the tree level (when the fast modes are not integrated over, but just ignored), the magnetic field is homogeneous and the magnetic flux is $q=2 k$. The vacuum wave functions can in this case be written explicitly, they are related ${ }^{3}$ to theta-functions of level $2 k$,

$$
\chi_{m} \sim \sum_{n=-\infty}^{\infty} \exp \left\{-2 \pi k\left(n+y+\frac{m}{2 k}\right)^{2}-2 \pi i k x y-4 \pi i k x\left(n+\frac{m}{2 k}\right)\right\},
$$

where

$$
x=\frac{C_{1} L}{(4 \pi)}, \quad y=\frac{C_{2} L}{(4 \pi)} .
$$

and $m=0, \ldots, 2 k-1$. When $k<0$, the vacuum wave functions are fermionic, involving the holomorphic factor $\psi$ (a superpartner of $C_{j}$ ). Not all of the states (1.9) are admissible. The gauge invariance of the states in the original theory dictates that the effective wave functions should be invariant under Weyl reflections. Indeed, such Weyl reflections for the effective wave functions can be realized as certain large gauge transformations for the wave functions of original theory. For $\mathrm{SU}(2)$, Weyl group involves only one element, $A_{j} \rightarrow-A_{j}$. There are $k+1$ Weyl invariant combinations:

$$
\Psi_{0}, \Psi_{k}, \text { and } \Psi_{m}+\Psi_{2 k-m}(m=1, \ldots, k-1) .
$$

\footnotetext{
${ }^{2}$ For other groups, we have the motion over $T \times T, T$ being the maximal torus of the group.

${ }^{3}$ We will explain how this comes about in section 4 , see eqs. (4.14)-(4.16).
} 
Thus, at the tree level, we obtain the value $k+1$ for the index.

This value is modified when taking into account loops. The loops are irrevant in the middle of the dual torus, $C_{j} \in(0,4 \pi / L)$, where the Born-Oppenheimer approximation works well, but there are four "corners" where the approximation breaks down and loop corrections are relevant:

$$
C_{j}=(0,0) ; \quad C_{j}=(2 \pi / L, 0) ; \quad C_{j}=(0,2 \pi / L) ; \quad C_{j}=(2 \pi / L, 2 \pi / L) .
$$

We have shown in ref. [2] that the fermion loop 4 brings about an extra effective magnetic field with the flux $-1 / 2$ in each corner and $\Phi^{\text {extra fermion }} /(2 \pi)=-2$ all together. (For $k<0$, the signs of both the tree-level and loop-induced fluxes are opposite.) This alone would renormalize the total flux $2 k \rightarrow 2 k-2$, which would give $(k-1)+1$ Weyl-invariant vacuum states in agreement with (1.7). However, in the framework of this approach, the gluon loop seems to be equally important. It gives twice as large extra effective magnetic flux as the fermion one, but with the opposite sign. This would give the total flux $2 k-2+4$ $=2(k+1)$ and $k+2$ vacuum states with a blatant contradiction with (1.7)!

The resolution of the paradox goes along the lines anticipated already in [2]. The extra flux comes from the regions around the singular points (the corners (1.11)) where the BornOppenheimer approximation breaks down. This makes the whole analysis precarious. The raison d'être for this paper are two simple remarks:

- The effective Lagrangian (as any Lagrangian) can be determined only up to a total time derivative. Normally, such time derivative does not change anything, but if we add a derivative $\sim \frac{d}{d t} \ln \left[\left(C_{1}+i C_{2}\right) /\left(C_{1}-i C_{2}\right)\right]$, which is singular in the corner $C_{j}=0$, this brings about an extra delta-functional flux, which may change the index. The effective action method does not control well such contributions, which leaves the result for the index uncertain.

- The corners can still be treated within the effective Hamiltonian method. To be quite precise, the ambiguity mentioned above displays itself also there and consists in the freedom to multiply the fast ground state wave function by a singular factor

$$
\sim\left[\left(C_{1}+i C_{2}\right) /\left(C_{1}-i C_{2}\right)\right]^{\alpha} .
$$

The point is, however, is that this ambiguity can be fixed by imposing proper boundary conditions at the corners. Indeed, in each such region, one can still apply the Born-Oppenheimer procedure, to single out a finite number of slow variables and integrate over all other variables. For example, in the region near the corner, $A_{j}=0$ the slow variables represent constant (not necessarily Abelian) Fourrier modes $A_{j}^{a=1,2,3(\mathbf{0})}$. The effective Hamiltonian (it is nothing but the original Hamiltonian dimensionally reduced to $(0+1)$ dimensions) involves, thus, 6 bosonic variables. 6 is greater that 2 , but, still, this problem turns out to be treatable, if capitalizing on the gauge invariance requirements.

\footnotetext{
${ }^{4}$ It is sufficient to consider a single loop. One can argue that the second and higher loops do not contribute.
} 
Matching the vacuum solutions of this corner effective Hamiltonian to the solution of the Abelian valley effective Hamiltonian (this is possible to do even though the former are not known exactly) gives us boundary conditions for the Abelian BO wave function and count the number of vacuum solutions. Our final result coincides with (1.7).

The rest of the paper is organised as follows. In the next section, we perform an accurate calculation of gluon loop contribution in the effective action. (In ref. [2], only the calculation of the fermion loop was described in details.) We show that, indeed,

$$
\Phi^{\text {extra gluon }}=-2 \Phi^{\text {extra fermion }} .
$$

We discuss then singular total derivative contributions that are difficult to control.

In section 3, we analyse the dimensionally reduced QM Hamiltonian near the corner $A_{j}=0$, study what happens with its vacuum wave function near the Abelian valley, $A_{j}^{a} \approx$ $C_{j} \delta^{a 1}$, and calculate the effective valley Hamiltonian. The latter involves PancharatnamBerry $(\mathrm{PB})$ phase $[13,14]$ - an extra gauge potential in the space $\left\{C_{j}\right\}$ of slow variables arising after integrating out the fast ones. We show that, using the most natural definition of what is understood under the effective wave function, this PB phase is associated only with the fermion factor in the fast wave function and brings about the contribution $-1 / 2$ to the flux from each corner.

In section 4, we show how, irrespectively of the ambiguity associated with including or not a factor like (1.12) in $\Psi^{\text {fast }}$, the requirement of regularity for the wave functions at the corners allows one to find them on the full dual torus and count them. Extra gauge fields dwelling in the corners modify both the form of the wave functions (they are now given by eq. (4.17) below) and their counting. Before imposing the Weyl invariance requirement, we have $2(k-1)$ rather than $2 k$ functions of which only $k$ are left when Weyl invariance is imposed.

There are four technical appendices. appendix A is purely mathematical being devoted to theta-functions. In appendix B, we remind how the states were counted in pure CS theory. In appendix C, we accurately study the behavior of the non-Abelian ground state wave functions near a corner. In appendix D, we construct the corner Hamiltonian with explicitly resolved gauge constraints.

\section{Effective action}

Let us discuss first the renormalization of the theory (1.1) in the infinite volume. It was studied earlier $[15,16]$ in covariant gauges. One can use, alternatively, the Hamilton gauge $A_{0}=0$, in which case the gluon propagator is

$$
D_{j k}^{a b}(\omega, \mathbf{p})=\frac{i g^{2} \delta^{a b}}{\omega^{2}-\mathbf{p}^{2}-m^{2}}\left[\delta_{j k}-\frac{p_{j} p_{k}}{\omega^{2}}-\frac{i m}{\omega} \epsilon_{j k}\right] .
$$

This choice simplifies the calculations in the gluon sector (no ghosts and only one graph to evaluate). The known result

$$
k \rightarrow k-\left(\frac{N}{2}\right)_{\text {ferm. loop }}+(N)_{\text {gluon loop }}=k+\frac{N}{2}
$$


is, of course, reproduced.

To evaluate the effective action in the small finite volume, we note first that the corrections are only large near one of the corners. If choosing, say, the region near $A_{j}=0$, we notice that one should only take into account the zero Fourrier modes of the gluon fields propagating in the loop - nonzero modes have masses $\sim 1 / L$ and their contribution is suppressed for small volumes. Thus, we can neglect the spatial dependence of the fields and perform the calculation in the dimensionally reduced theory with the Lagrangian

$$
\begin{aligned}
L= & \frac{1}{2 g_{0}^{2}}\left(\dot{A}_{j}^{a}\right)^{2}+\frac{m}{2 g_{0}^{2}} \epsilon_{j k} \dot{A}_{j}^{a} A_{k}^{a}-\frac{1}{4 g_{0}^{2}}\left[\left(A_{j}^{a} A_{j}^{a}\right)^{2}-A_{j}^{a} A_{k}^{a} A_{j}^{b} A_{k}^{b}\right] \\
& -i \frac{\epsilon^{a b c}}{2}\left[\bar{\psi}^{a} \bar{\psi}^{b} A_{+}^{c}+\psi^{a} \psi^{b} A_{-}^{c}\right]+m \bar{\psi}^{a} \psi^{a},
\end{aligned}
$$

where

$$
g_{0}^{2} \equiv g_{1 d}^{2}=\frac{g_{3 d}^{2}}{L^{2}}
$$

and $A_{ \pm}^{a}=A_{1}^{a} \pm i A_{2}^{a}$ (and similarly for other vectors below). We assume the Abelian background to be directed along the first color axis, $A_{j}^{1} \equiv C_{j}$. Then $A_{j}^{a=2,3}$ are the fluctuations. An inspection of the quadratic in $A_{j}^{a=2,3}$ part of the Lagrangian,

$$
g_{0}^{2} L^{\text {fast }}=\frac{1}{2}\left(\dot{A}_{j}^{a}\right)^{2}+\frac{m}{2} \epsilon_{j k} \dot{A}_{j}^{a} A_{k}^{a}-\frac{1}{2}\left(A_{j}^{a} A_{k}^{a}\right)\left(\mathbf{C}^{2} \delta_{j k}-C_{j} C_{k}\right)+\text { other terms },
$$

gives the QM propagator

$$
D_{j k}^{a b}=\frac{i g_{0}^{2} \delta^{a b}}{\omega^{2}-\mathbf{C}^{2}-m^{2}}\left[\delta_{j k}-\frac{C_{j} C_{k}}{\omega^{2}}-\frac{i m}{\omega} \epsilon_{j k}\right]
$$

(it is obtained from the field theory propagator (2.1) by replacing $\mathbf{p} \rightarrow \mathbf{C}$, dividing by $L^{2}$ and assuming $a, b=2,3)$.

We are hunting for the corrections $\sim \dot{C}_{j} \mathcal{A}_{j}(\mathbf{C})$ in the effective Lagrangian. ${ }^{5}$ To this end, we should pose

$$
\mathbf{C}(\tau) \rightarrow \mathbf{C}+\mathbf{E} \tau
$$

( $\tau$ is Euclidean time; to evaluate the graphs, we are going to perform, as usual, the Wick rotation etc.) and proceed in the same way as in the appendix of the previous paper [2] where the calculation of the fermion loop was described in details.

We just quote here the result of that calculation. The fermion loop contribution to the effective Lagrangian can be represented as

$$
\Delta^{F} L_{\mathrm{eff}}=-E_{j} \mathcal{A}_{j}^{F}(\mathbf{C})
$$

with

$$
\mathcal{A}_{j}^{F}(\mathbf{C})=\frac{\epsilon_{j k} C_{k}}{2 \mathbf{C}^{2}}\left[1-\frac{m}{\sqrt{m^{2}+\mathbf{C}^{2}}}\right] .
$$

\footnotetext{
${ }^{5}$ Do not confuse curly $\mathcal{A}_{j}$ with the physical gauge potentials $A_{j}$. The former are the functions of the latter!
} 


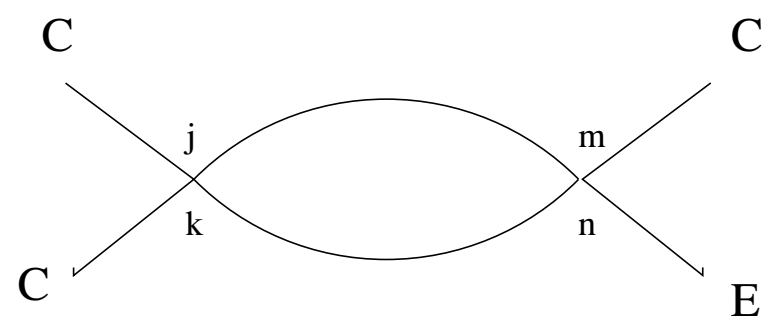

Figure 1. The lowest order gluon loop contribution in $L_{\text {eff }}$.

The corresponding magnetic field is

$$
\mathcal{B}^{F}=\epsilon_{j k} \partial_{j} \mathcal{A}_{k}^{F}=-\frac{m}{2\left(\mathbf{C}^{2}+m^{2}\right)^{3 / 2}}
$$

It has the flux

$$
q^{F}=\frac{\Phi^{F}}{2 \pi}=\frac{1}{2 \pi} \int \mathcal{B}^{F}(\mathbf{C}) d \mathbf{C}=-\frac{1}{2}
$$

In the bosonic case, the lowest order (in the background) contribution is $\sim E C C C$ and is described by the graph in figure 1.

The calculation gives

$$
\begin{aligned}
L_{\text {eff }}^{\text {figure } 1} & =\left.\frac{1}{4 g_{0}^{4}}\left(\mathbf{C}^{2} \delta_{j k}-C_{j} C_{k}\right)\left[2(\mathbf{C E}) \delta_{m n}-C_{n} E_{m}-C_{m} E_{n}\right] \int_{-\infty}^{\infty} \frac{d \omega}{2 \pi} D_{j m}^{a b}(\omega) \frac{\partial}{\partial \omega} D_{n k}^{a b}(\omega)\right|_{\mathbf{C}=0} \\
& =-2 m \mathbf{C}^{2} \epsilon_{j m} C_{j} E_{m} \int_{-\infty}^{\infty} \frac{d \Omega}{2 \pi\left(\Omega^{2}+m^{2}\right)^{3}}=-\frac{3}{8 m^{4}} \mathbf{C}^{2} \epsilon_{j k} C_{j} E_{k} .
\end{aligned}
$$

The effective Lagrangian accepts also contribution $\sim E C C C C C$ from the six-leg graphs, etc. To sum them all up, one should (see ref. [2] to understand why)

- Write the expression (2.12) and restore the dependence on $\mathbf{C}$ in Green's functions. This gives a phantasy effective Lagrangian.

- The true effective Lagrangian is obtained by multiplying the term $\propto E C^{2 n+1}$ in the expansion of $L_{\text {eff }}^{\text {phant }}$ by $2 /(n+1)$.

This finally gives

$$
\begin{aligned}
\mathcal{A}_{j}^{B}(\mathbf{C}) & =-8 m \epsilon_{j k} C_{k} \int_{0}^{1} s^{3} d s \int \frac{d \omega}{2 \pi\left(\omega^{2}+s^{2} \mathbf{C}^{2}+m^{2}\right)^{3}} \\
& =-\frac{\epsilon_{j k} C_{k}}{2 \mathbf{C}^{2}}\left[2-\frac{3 m}{\sqrt{m^{2}+\mathbf{C}^{2}}}+\frac{m^{3}}{\left(m^{2}+\mathbf{C}^{2}\right)^{3 / 2}}\right] .
\end{aligned}
$$

The corresponding magnetic field is

$$
\mathcal{B}^{B}(\mathbf{C})=\frac{3 m \mathbf{C}^{2}}{2\left(\mathbf{C}^{2}+m^{2}\right)^{5 / 2}} .
$$


Its flux is

$$
q^{B}=1=-2 q^{F} .
$$

However, as was already emphasized in the Introduction, this effective action calculation cannot be trusted because:

- By its very meaning (relying on the smallness of fluctuations with respect to the background), it makes sense only when $\mathbf{C} \neq 0$.

- One can always add to the Lagrangian a total derivative. In our case, we can add a total derivative that is singular at $\mathbf{C}=0$. In particular, one can add the derivative $\frac{-i}{2} \frac{d}{d t} \ln \left(C_{+} / C_{-}\right)$which leads to the extra contribution

$$
\mathcal{A}_{j}=\epsilon_{j k} \frac{C_{k}}{\mathbf{C}^{2}}
$$

in the effective vector potential. The calculation described above cannot "detect" this singular piece - by construction, the vector potential (2.13) is analytic at $\mathbf{C}=0$.

The contribution (2.16) gives a delta-singular effective magnetic field with the flux -1 , which would exactly cancel the gluon loop contribution.

\section{Effective Hamiltonian}

The important message that we want to convey here is that one can resolve this ambiguity, if using the Hamiltonian rather than Lagrangian language and matching the effective wave function on the Abelian moduli space to the wave function in the vicinity of the origin.

We will proceed in the classical Born-Oppenheimer spirit, subdivide all the variables into slow variables relevant to the low-energy dynamics and the fast ones to be integrated over. Explicitly, we represent the full wave function as

$$
\Psi_{\text {low energy }}\left(x^{\text {fast }}, x^{\text {slow }}\right) \approx \chi^{\text {eff }}\left(x^{\text {slow }}\right) \Psi_{0}\left(x^{\text {fast }}\right),
$$

with $\Psi_{0}\left(x^{\text {fast }}\right)$ being the ground state of the fast Hamiltonian where the slow variables play the role of parameters. Then the effective Hamiltonian that acts on $\chi^{\text {eff }}\left(x^{\text {slow }}\right)$ represents the average of the full Hamiltonian over the fast vacuum state,

$$
\hat{H}^{\mathrm{eff}}=\langle\hat{H}\rangle_{\text {fast vacuum }} .
$$

This method was used in [9] for non-chiral $(3+1)$ supersymmetric gauge theories. The leading order effective Hamiltonian describes in this case just the free motion over $T \times T \times T$ ( $T$ being the maximal torus of the group) with an additional Weyl invariance requirement imposed on the states. In $[17,18]$, we applied this method for chiral $(3+1)$ theories with left-right asymmetric matter content. In this case, nontrivial PB phases appear. For example, in the chiral SQED with 8 left chiral matter multiplets of charge 1 and a right chiral multiplet of charge 2 , the motion runs over $T^{3}$ equipped with a magnetic monopole of charge +7 and 7 monopoles of charge -1 . The method can be (and was $[19,20]$ ) extended 
such that loop corrections to any BO order in the effective Hamiltonian can be calculated, but it suffices for us here to stay in the approximation (3.1) and to evaluate (3.2).

For gauge theores, the Schrödinger equation should be supplemented by the Gauss law constraints. The latter can be treated either as quantum constraints to be imposed on the states, or else one can resolve the constraints at the classical level such that only gauge-invariant variables are left in the Hamiltonian. The former method is simpler and we use it in the main text. For methodic purposes, we repeated the analysis with the gauge constraints explicitly resolved, and this is the subject of appendix D. ${ }^{6}$

As was discussed above, we are basically interested only in the dynamics in one of the corners of the dual torus where the extra contributions to the flux come from. In that case, higher Fourrier modes are irrelevant and we are in a position to study the dimensionally reduced SQM theory. The quantum supercharges in this reduced theory are

$$
g_{0} Q=E_{-}^{a} \psi^{a}+i B^{a} \bar{\psi}^{a}, \quad g_{0} \bar{Q}=\bar{\psi}^{a} E_{+}^{a}-i B^{a} \psi^{a},
$$

where $g_{0}$ is the QM coupling constant (2.4) of canonical dimension $m^{3 / 2}$ and

$$
E_{j}^{a}=g_{0}^{2} \Pi_{j}^{a}-\frac{m}{2} \epsilon_{j k} A_{k}^{a}
$$

with $\Pi_{j}^{a}=-i \partial / \partial A_{j}^{a}$. The fermion variables are expressed via the constant modes of the original field theory variables in eq. (1.1) as

$$
\begin{aligned}
\psi^{a} & =\frac{\lambda_{1}^{(\mathbf{0})}-i \lambda_{2}^{(\mathbf{0})}}{g_{0} \sqrt{2}}, \\
\bar{\psi}^{a} & =\frac{\lambda_{1}^{(\mathbf{0})}+i \lambda_{2}^{(\mathbf{0})}}{g_{0} \sqrt{2}} .
\end{aligned}
$$

In holomorphic representation, $\bar{\psi}^{a} \equiv \partial / \partial \psi^{a}$. Finally,

$$
B^{a}=\frac{1}{2} \epsilon^{a b c} \epsilon_{j k} A_{j}^{b} A_{k}^{c}=-\frac{i}{2} \epsilon^{a b c} A_{-}^{b} A_{+}^{c}
$$

is the non-Abelian magnetic field strength. One can derive $Q^{2}=A_{-}^{a} G^{a}$, where

$$
G^{a}=\epsilon^{a b c}\left(A_{j}^{b} \Pi_{j}^{c}-i \psi^{b} \bar{\psi}^{c}\right)
$$

is the Gauss law. $Q$ and $\bar{Q}$ are thus nilpotent in the Hilbert space involving only gauge unvariant states. The anticommutator $\{Q, \bar{Q}\} / 2$ gives the Hamiltonian,

$$
\begin{aligned}
H= & \frac{g_{0}^{2}}{2}\left(\Pi_{j}^{a}-\frac{m}{2 g_{0}^{2}} \epsilon_{j k} A_{k}^{a}\right)^{2}+\frac{1}{4 g_{0}^{2}}\left[\left(A_{j}^{a} A_{j}^{a}\right)^{2}-A_{j}^{a} A_{k}^{a} A_{j}^{b} A_{k}^{b}\right] \\
& +i \frac{\epsilon^{a b c}}{2}\left[\bar{\psi}^{a} \bar{\psi}^{b} A_{+}^{c}+\psi^{a} \psi^{b} A_{-}^{c}\right]+\frac{m}{2}\left(\psi^{a} \bar{\psi}^{a}-\bar{\psi}^{a} \psi^{a}\right)
\end{aligned}
$$

One can make three simple observations.

\footnotetext{
${ }^{6}$ For sure, such an analysis is not technicably possible in a gauge field theory. But in a gauge quantum mechanics, it is quite feasible.
} 
- The Hamiltonian (3.8) involves two dimensionfull parameters, $g_{0}$ and $m$. They are ordered as

$$
g_{0}^{2} \gg m^{3}
$$

This is a corollary of the condition for the box to be small, $m L \ll 1$. As a result, the mass terms in (3.8) are smaller than the terms without mass.

- The Hamiltonian admits an integral of motion - the angular momentum

$$
j=\epsilon_{j k} A_{j}^{a} \Pi_{k}^{a}+\frac{1}{2} \psi^{a} \bar{\psi}^{a} .
$$

The eigenvalues of $j$ are integer for bosonic states and half-integer for fermionic states. Note that $j$ does not commute with the supercharges (3.3), such that a sector with definite $j$ is not supersymmetric.

- On the other hand, the Hamiltonian (3.8) does not preserve the fermion charge. ${ }^{7}$ That means that eigenfunctions of (3.8) do not have a definite fermion charge. The bosonic states represent a superposition of the terms of charge $F=0$ and $F=2$,

$$
\Psi=P+\frac{1}{2} \epsilon^{a b c}\left(S A_{-}^{a}+R A_{+}^{a}+T B^{a}\right) \psi^{b} \psi^{c},
$$

with the scalar functions $P, S, R, T$ depending only on three gauge-invariant variables $\mathcal{X}=A_{+}^{a} A_{-}^{a}, \mathcal{Z}=A_{+}^{a} A_{+}^{a}$ and $\overline{\mathcal{Z}}=A_{-}^{a} A_{-}^{a}$. The wave functions (3.11), (3.12) are the eigenstates of the operator (3.10). This means that $P, S$ are transformed in the same way under rotations, while $T$ has the extra charge -1 and $R$ - the extra charge -2 . Likewise, the fermion states represent mixtures of the $F=1$ and $F=3$ components,

$$
\Psi=\frac{1}{6} P^{\prime} \epsilon^{a b c} \psi^{a} \psi^{b} \psi^{c}+\left(S^{\prime} A_{+}^{a}+R^{\prime} A_{-}^{a}+T^{\prime} B^{a}\right) \psi^{a} .
$$

Again, $P^{\prime}$ and $S^{\prime}$ have the same charges, $T^{\prime}$ has the extra charge +1 and $R^{\prime}-$ the extra charge +2 .

To study the behavior of this system at the vicinity of the Abelian valley, it is convenient to subdivide six bosonic variables $A_{j}^{a}$ into:

- two Abelian slow variables $C_{j} \equiv A_{j}^{1}$,

- fast variables $A_{j}^{a=2,3} \equiv b_{j}^{a}$ which in turn involve i) two projections $b_{j}^{a} C_{j}$ - the gauge degrees of freedom describing color rotations $A_{j}^{1} \rightarrow A_{j}^{2,3}$, ii) After the partial gauge fixing $b_{j}^{a} C_{j}=0$, we are left with two remaining degrees of freedom, which include a physical gauge-invariant fast fluctuation variable $b^{2}=\left(b_{j}^{a}\right)^{2}$ and an unfixed yet gauge angle describing the rotation around the first color axis (the chosen direction for the slow background).

\footnotetext{
${ }^{7}$ It shares this feature with the Hamiltonian of $\mathcal{N}=44 d$ SYM theory while, in $\mathcal{N}=14 d$ theories, the fermion charge is concerved [21].
} 
The slow variables $C_{j}$ should lie in the range

$$
g_{0}^{2 / 3} \ll|\mathbf{C}| \equiv a \ll \frac{g_{0}}{\sqrt{m}} .
$$

The lower bound here is the scale at which the characteristic values of $b$ becomes comparable to $a$ such that the $\mathrm{BO}$ approximation is no longer valid. The upper bound corresponds to $a \sim 1 / L$. This corresponds to the interior of the dual torus where higher Fourrier harmonics (that we neglect) begin to play an important role.

In supersymmetric theory, the separation of fast and slow variables is more convenient to perform at the level of supercharges rather than for the Hamiltonian. The leading in the $\mathrm{BO}$ parameter $\sim b / a$ part of the supercharges (3.3) can be represented as

$$
\begin{aligned}
2 Q^{\mathrm{fast}} & =\frac{g_{0}}{C_{+}}\left(C_{+} \Pi_{-}^{a}-C_{-} \Pi_{+}^{a}\right) \psi^{a}+\frac{1}{g_{0}} \epsilon^{a b} \bar{\psi}^{a}\left(C_{+} b_{-}^{b}-C_{-} b_{+}^{b}\right), \\
2 \bar{Q}^{\mathrm{fast}} & =\frac{g_{0}}{C_{-}}\left(C_{-} \Pi_{+}^{a}-C_{+} \Pi_{-}^{a}\right) \bar{\psi}^{a}+\frac{1}{g_{0}} \epsilon^{a b} \psi^{a}\left(C_{-} b_{+}^{b}-C_{+} b_{-}^{b}\right)
\end{aligned}
$$

with $a, b=2,3$. When deriving (3.14), we were allowed to replace

$$
\Pi_{-}^{a} \rightarrow \frac{1}{2}\left(\Pi_{-}^{a}-\frac{\Pi_{+}^{a} C_{-}}{C_{+}}\right), \quad \Pi_{+}^{a} \rightarrow \frac{1}{2}\left(\Pi_{+}^{a}-\frac{\Pi_{-}^{a} C_{+}}{C_{-}}\right)
$$

bearing in mind that the Hilbert space where the fast supercharges act involves wave functions not depending on the projections $b_{j}^{a} C_{j}$ such that $C_{j} \Pi_{j}^{a} \Psi=0$. If we also require the wave functions to be annihilated by $\hat{G}^{1}=\epsilon^{a b}\left(b_{j}^{a} \Pi_{j}^{b}-i \psi^{a} \bar{\psi}^{b}\right)$, the supercharges (3.14) become nilpotent.

The corresponding fast Hamiltonian is ${ }^{8}$

$$
H^{\mathrm{fast}}=\frac{g_{0}^{2}}{2} \Pi_{+}^{a} \Pi_{-}^{a}-\frac{1}{8 g_{0}^{2}}\left(C_{+} b_{-}^{a}-C_{-} b_{+}^{a}\right)^{2}+\frac{i}{2} \epsilon^{a b}\left(C_{+} \bar{\psi}^{a} \bar{\psi}^{b}+C_{-} \psi^{a} \psi^{b}\right) .
$$

The Hamiltonian (3.15) represents a variety of supersymmetric oscillator. It has a single bosonic ground state. Up to a numerical factor, its wave function is

$$
\Psi_{0}^{\mathrm{fast}}=\left(C_{+} C_{-}\right)^{-1 / 4}\left[2 i+\sqrt{\frac{C_{-}}{C_{+}}} \epsilon^{a b} \psi^{a} \psi^{b}\right] \exp \left\{\frac{1}{8 g_{0}^{2} \sqrt{C_{-} C_{+}}}\left(C_{+} b_{-}^{a}-C_{-} b_{+}^{a}\right)^{2}\right\}
$$

This is basically a product of eq. (2.28) and eq. (2.30) in ref. [2] with $m$ set to zero ${ }^{9}$ and generalized to an arbitrary $C_{j} \neq C \delta_{j 1}$. We have included the factor $\left(C_{-} C_{+}\right)^{-1 / 4}$ in the definition of $\Psi_{0}^{\text {fast }}$ for the normalization integral

$$
\int d x^{\text {fast }}\left|\Psi_{0}^{\text {fast }}\right|^{2}
$$

\footnotetext{
${ }^{8}$ Cf. refs. [22, 23] where a similar fast Hamiltonian for the quantum mechanics derived from $\mathrm{N}=44 d$ SYM theory was written and discussed.

${ }^{9}$ It is more consistent not to include the mass terms when writing the fast supercharges and the Hamiltonian, because they are suppressed compared to the others (see the comment after eq. (3.8)). We emphasize that, while, in the Feynman graph method addressed in the previous section, we were obliged to include the mass terms in the propagators to regularize infrared singularities, we do not need to bother about mass in the Hamiltonian approach.
} 
with $^{10}$

$$
d x^{\mathrm{fast}} \sim\left(C_{+} C_{-}\right)^{2} d_{\text {fermions }} d^{2} b_{+} d^{2} b_{-} \prod_{a=2,3} \delta\left(C_{+} b_{-}^{a}+C_{+} b_{+}^{a}\right)
$$

not to depend on $C_{j}$.

Note that the fermion factor in (3.16) involves only two terms, not four terms as in a generic decomposition (3.11). That is because, at the valley, there is no difference between 3 bifermion structures in (3.11). They are expressed into one another by multiplying over a proper function of slow variables $C_{ \pm}$.

With the fast ground state wave function in hand, we can determine the effective Hamiltonian. Assume first $k>0$. By analyzing the effective Hamiltonian in the interior of the dual torus, we have seen that the vacuum states are in this case bosonic (see eq. (1.9)). This should concern also the Hamiltonian (3.8) describing the "corner dynamics". Thus, the vacuum wave function annihilated by the full supercharges (3.3) has the form (3.11). Consider this function and the equations $\hat{Q} \Psi=\hat{\bar{Q}} \Psi=0$ near the Abelian valley (3.13). The wave function there is approximately given by the product [cf. eq. (3.1)]

$$
\Psi_{0}=\chi^{\mathrm{eff}}\left(C_{j}\right) \Psi_{0}^{\mathrm{fast}}
$$

with $\Psi_{0}^{\text {fast }}$ written in (3.16). The effective supercharges acting on $\chi^{\text {eff }}\left(C_{j}\right)$ are

$$
Q^{\mathrm{eff}}=\langle\Delta Q\rangle_{0}, \quad \bar{Q}^{\mathrm{eff}}=\langle\Delta \bar{Q}\rangle_{0}
$$

with

$$
\begin{aligned}
& \Delta Q=Q-Q^{\text {fast }}=-i \psi^{1}\left(2 g_{0} \frac{\partial}{\partial C_{+}}+\frac{m}{2 g_{0}} C_{-}\right)+\frac{\bar{\psi}^{1}}{2 g_{0}} \epsilon^{a b} b_{-}^{a} b_{+}^{b}-\frac{i m}{2 g_{0}} \psi^{a} b_{-}^{a}, \\
& \Delta \bar{Q}=\bar{Q}-\bar{Q}^{\text {fast }}=-i \bar{\psi}^{1}\left(2 g_{0} \frac{\partial}{\partial C_{-}}-\frac{m}{2 g_{0}} C_{+}\right)-\frac{\psi^{1}}{2 g_{0}} \epsilon^{a b} b_{-}^{a} b_{+}^{b}+\frac{i m}{2 g_{0}} \bar{\psi}^{a} b_{+}^{a} .
\end{aligned}
$$

Two last terms give zero after averaging. We obtain

$$
\begin{aligned}
Q^{\mathrm{eff}} & =-i \psi^{1}\left(2 g_{0} \frac{\partial}{\partial C_{+}}+\frac{m}{2 g_{0}} C_{-}\right)-2 i g_{0} \psi^{1}\left\langle\frac{\partial}{\partial C_{+}}\right\rangle_{0}, \\
\bar{Q}^{\mathrm{eff}} & =-i \bar{\psi}^{1}\left(2 g_{0} \frac{\partial}{\partial C_{-}}-\frac{m}{2 g_{0}} C_{+}\right)-2 i g_{0} \bar{\psi}^{1}\left\langle\frac{\partial}{\partial C_{-}}\right\rangle_{0} .
\end{aligned}
$$

The last terms above involve PB phases,

$$
\mathcal{A}_{-}^{\mathrm{PB}} \propto\left\langle\frac{\partial}{\partial C_{+}}\right\rangle_{0}=-\frac{1}{4 C_{+}}, \quad \mathcal{A}_{+}^{\mathrm{PB}} \propto\left\langle\frac{\partial}{\partial C_{-}}\right\rangle_{0}=\frac{1}{4 C_{-}}
$$

and hence

$$
\mathcal{A}_{j}^{\mathrm{PB}} \propto \frac{\epsilon_{j k} C_{k}}{\mathbf{C}^{2}} .
$$

\footnotetext{
${ }^{10}$ This particular form of $d x^{\text {fast }}$, in particular the important factor $\left(C_{+} C_{-}\right)^{2}$ there follows from the requirement that the original measure $\prod_{a j} d A_{j}^{a}$ goes over to $d C_{+} d C_{-} d x^{\text {fast }}$ on the valley. See also eq. (D.3).
} 
Note that the averages (3.22) depend only on the fermion factor in the fast wave function (3.16), the bosonic factor does not produce any phases. Indeed, nontrivial PB phases

$$
\mathcal{A}_{j}^{\mathrm{PB}} \propto \frac{\int\left(\Psi_{0}^{\mathrm{fast}}\right)^{*} \frac{\partial}{\partial C_{j}} \Psi_{0}^{\text {fast }} d x^{\text {fast }}}{\int\left(\Psi_{0}^{\text {fast }}\right)^{*} \Psi_{0}^{\text {fast }} d x^{\text {fast }}}
$$

can arise only due to complexities in the wave function. The real bosonic exponential factor could contribute a total gradient in $\mathcal{A}_{j}^{\mathrm{PB}}$ ( which could then be eliminated by a gauge transformation), but this gradient is just absent, if choosing the normalization factor as in (3.16).

Thus, the effective supercharges involve tree level vector potentials $\mathcal{A}_{ \pm}^{\text {tree }} \propto \pm k C_{ \pm}$ (these are vector potentials for the constant magnetic field on the dual torus of total flux $2 k)$ and the induced potentials (3.22). The latter have the form (2.16) with the factor $1 / 2$. These potentials have a delta-functional magnetic field. ${ }^{11}$ One can be convinced that the corresponding flux is equal to $-1 / 2$, which coincides with the flux brought about by the fermion loops in the effective action method. On the other hand, there is no trace of the gluon loop contribution in the Hamiltonian approach!

Consider now the case $k<0$. The ground states are now fermionic having the form (3.12). In the vicinity of the valley, the wave function is natural to represent as

$$
\Psi_{0}=\psi^{1} \chi^{\text {eff }}\left(C_{j}\right) \tilde{\Psi}_{0}^{\text {fast }}
$$

with

$$
\tilde{\Psi}_{0}^{\mathrm{fast}}=\sqrt{\frac{C_{+}}{C_{-}}} \Psi_{0}^{\mathrm{fast}} .
$$

The appearance of the extra factor $\sqrt{C_{+} / C_{-}}$in (3.26) reflects the presence of the factor $A_{+}^{a}$ in the second term in (3.12) rather than the factor $A_{-}^{a}$ in the second term in (3.11). (As was discussed above, in the vicinity of Abelian valley, two other fermion bilinear terms are reduced to the term $\propto S A_{-}^{a}$ in the bosonic case and to the term $\propto S^{\prime} A_{+}^{a}$ in the fermionic case. For less heuristic justification of the choice (3.26), see the footnote after (4.2) below.)

When transferring the analysis above to the case $k<0$ with the modified fast vacuum function (3.26), we obtain the induced singular vector potentials like in (2.16) with the positive $\delta$-functional flux $\Delta \Phi_{k<0} /(2 \pi)=1 / 2$ ( the contribution $-1 / 2$ coming from $\Psi_{0}^{\text {fast }}$, as above and the contribution +1 from the factor $\sqrt{C_{+} / C_{-}}$.

The sign of the induced flux is thus always opposite to the tree-level flux. Recalling that the full dual torus includes four singular points, one obtains the flux renormalization $2 k \rightarrow 2(k-1)$ in the case $k>0$ and $2 k \rightarrow 2(k+1)$ in the case $k<0$. This finally gives the answer (1.7) for the index.

At this stage, our findings have the flavour of a paradox. Indeed, we discovered in the previous section that the effective Lagrangian calculations involve an intrinsic ambiguity

\footnotetext{
${ }^{11}$ This is so for zero mass. When $m \neq 0$, the flux is concentrated in the region $a \sim m$, which is much smaller than the lower bound in (3.13).
} 
associated with adding a singular total derivative. Now we are claiming to resolve this ambiguity in the Hamiltonian approach. But the Lagrangian and Hamiltonian descriptions must be completely equivalent. How come?

The resolution of this paradox is the subject of the next section.

\section{Torus with the corners}

Note first of all that there is an ambiguity also in the Hamiltonian method that exactly corresponds to the Lagrangian ambiguity mentioned above. One is always allowed to introduce a factor $\left(C_{+} / C_{-}\right)^{\alpha}$ in the fast vacuum wave function - the slow variables $C_{j}$ enter as parameters in the fast Hamiltonian and we cannot decide whether to include this factor in the definition of $\Psi_{0}^{\text {fast }}$ or not. This uncertainty translates into the uncertainty of the coefficient of the $\delta$-functional flux located at the points where the BO approximation breaks down. ${ }^{12}$

In particular, choosing $\alpha=1 / 2$ effectively brings about the additional unit flux in the origin. In the full problem, this amounts to adding four units of flux (one in each corner), which exactly imitates the gluon loop contribution.

This ambiguity cannot be resolved while staying on the Abelian valley. Our main point is, however, that it can be fixed if imposing the additional requirement for the wave function of the full QM Hamiltonian (3.8) to be regular at the origin $A_{j}^{a}=0$.

Unfortunately, right near the corner where the Abelian BO approximation breaks down, the equation $Q \Psi=0$ cannot be solved analytically. Still, we can approach the corner from the Abelian valley side. Consider the effective supercharge (3.21). Bearing in mind (3.22), it is proportional to

$$
Q^{\mathrm{eff}} \propto \frac{\partial}{\partial C_{+}}-\frac{1}{4 C_{+}}+\frac{m}{4 g_{0}^{2}} C_{-} .
$$

A generic solution to the equation $Q^{\mathrm{eff}} \chi\left(C_{k}\right)=0$ is

$$
\chi\left(C_{k}\right) \sim\left(C_{-} C_{+}\right)^{1 / 4} P\left(C_{-}\right) \exp \left\{-\frac{m C_{-} C_{+}}{4 g_{0}^{2}}\right\}
$$

with an arbitrary entire function $P\left(C_{-}\right)$. In the range (3.13), the exponential factor in eq. (4.2) is close to 1 and irrelevant. ${ }^{13}$ An eigenstate with a definite angular momen-

\footnotetext{
${ }^{12}$ The ambiguity of this kind can appear only in a (2+1)-dimensional problem. In chiral (3+1)-dimensional theories, PB phases are unambigously fixed (up to a gauge transformation) $[17,18]$. Indeed, the induced field there has not the form of flux lines, but rather of a magnetic monopoles with a nonzero magnetic field strength not only at the origin, but also in its vicinity. It cannot be mimicked neither by a total derivative in the effective Lagrangian, nor by a factor entering the definition of the fast ground state wave function.

${ }^{13}$ When $k<0$, the effective wave function involves the factor $\psi^{1}$. It is annihilated automatically by $Q$,
} while

$$
\bar{Q}^{\mathrm{eff}} \propto \frac{\partial}{\partial C_{-}}-\frac{1}{4 C_{-}}
$$

and hence

$$
\chi\left(C_{k}\right) \sim \psi^{1}\left(C_{-} C_{+}\right)^{1 / 4} P\left(C_{+}\right)
$$


tum (3.10), or rather its effective counterpart in the sector $F=0$,

$$
\hat{j}^{\mathrm{eff}}=-i \epsilon_{j k} C_{j} \frac{\partial}{\partial C_{k}},
$$

behaves at the origin as

$$
\chi_{j}\left(C_{k}\right) \sim\left(C_{-} C_{+}\right)^{1 / 4} C_{-}^{-j} .
$$

If we require for $\chi_{j}\left(C_{k}\right)$ to be nonsingular at the origin, $j$ must be negative integer or zero. A glance at (3.16) tells us that, for $j \leq 0$, also the full wave function (3.18) behaves as

$$
\Psi_{0} \sim a^{-j}
$$

and is nonsingular. And, for positive $j$, it is singular. Such solutions should be excluded. ${ }^{14}$

Suppose now that we redefined the fast wave function according to

$$
\tilde{\Psi}_{0}^{\mathrm{fast}}=\sqrt{\frac{C_{+}}{C_{-}}} \Psi_{0}^{\mathrm{fast}} .
$$

The effective supercharge behaves now at the origin as

$$
\tilde{Q}^{\mathrm{eff}} \propto \frac{\partial}{\partial C_{+}}+\frac{1}{4 C_{+}} .
$$

The effective angular momentum operator is also modified,

$$
\hat{j}^{\mathrm{eff}}=-i \epsilon_{j k} C_{j} \frac{\partial}{\partial C_{k}}+1
$$

An eigenfunction of (4.10) with a definite value of $j$ is

$$
\tilde{\chi}_{j}\left(C_{k}\right) \sim\left(C_{-} C_{+}\right)^{-1 / 4} C_{-}^{1-j} .
$$

Again, for $\tilde{\chi}\left(C_{k}\right)$ to be nonsingular, the condition $j \leq 0$ should be satisfied. When multiplied by $\tilde{\Psi}_{0}^{\text {fast }}$ with the factor $\sqrt{C_{+} / C_{-}}$it now includes, we obtain the same full wave function (3.18) as before.

In other words, it does not matter at all whether the contribution of gluon loops is taken into account or not. What is important is to pose proper boundary conditions in the corners of the torus. Having done that, we are able to count the states and evaluate the index.

if the fast wave function is chosen as in (3.26). An eigenstate with a definite (half-integer in this case) $j$ behaves at the origin as

$$
\chi_{j}\left(C_{k}\right) \sim \psi^{1}\left(C_{-} C_{+}\right)^{1 / 4} C_{+}^{j-1 / 2},
$$

which is regular when $j \geq 1 / 2$.

${ }^{14}$ The representation (3.18) holds only in the valley approximation and, strictly speaking, we are not allowed to go with it right into the origin. It happens, however, that, irrespectively of whether the Abelian BO approximation is valid or not, one can follow the Abelian valley up to the very origin and rigourously prove that nonsingular at the origin wave function excludes positive $j$. This proof is the subject of appendix C. 
Let us first remind how it is done at the tree level without yet taking any loop (fermion or gluon) into account.

The equation $Q^{\text {eff }} \chi=0$ boils down in this case to

$$
\left(\frac{\partial}{\partial z}+\pi k \bar{z}\right) \chi=0
$$

$(z=x+i y$ with $x, y$ being defined in (1.10)). A generic solution to eq. (4.13) is

$$
\chi=e^{-\pi k \bar{z} z} F(\bar{z})
$$

where $F(\bar{z})$ is any antiholomorphic function. The particular solutions (1.9) are obtained if imposing proper boundary conditions $[2,24]$

$$
\begin{aligned}
& \chi(x+1, y)=e^{-2 \pi i k y} \chi(x, y), \\
& \chi(x, y+1)=e^{2 \pi i k x} \chi(x, y) .
\end{aligned}
$$

They can be represented as

$$
\chi_{m}=e^{-\pi k \bar{z} z} e^{\pi k \bar{z}^{2}} Q_{m}^{2 k}(\bar{z})
$$

in the notations of appendix A. A kinship of the wave functions (4.16) to the wave functions (B.9) of the pure CS states is clearly seen. It is the same kinship as between the wave functions of the lowest Landau levels and the wave functions of the states in the topological theory with the Lagrangian $\sim B \epsilon_{j k} \dot{x}_{j} x_{k}$.

Now, take loops into account. Call for definiteness $\chi\left(C_{k}\right)$ the coefficient of the fast wave function (3.16). Then, as we have seen, singular fluxes $\Phi /(2 \pi)=-1 / 2$ are added in each corner. The gauge field due to each such flux line is a singular pure gauge, like in eq. (2.16). This brings about a factor $\sim\left(C_{+} / C_{-}\right)^{1 / 4} \rightarrow(z / \bar{z})^{1 / 4}$ in the corner $z=0$ and, similarly, in the other corners. Thus, the effective nonsingular wave functions satisfying the boundary conditions (4.15) have the form

$$
\chi_{m}=e^{-\pi k \bar{z} z+\pi k \bar{z}^{2}} \prod_{n p}\left(\frac{z+n / 2+i p / 2}{\bar{z}+n / 2-i p / 2}\right)^{1 / 4} Q_{m}^{2 k-2}(\bar{z}) \sqrt{Q_{3}^{4}(\bar{z})-Q_{1}^{4}(\bar{z})},
$$

where the product runs over all integer $n, p$. The argument of the square root is the function (B.12) with four zeros in the four corners. The square root has branching points at the corners, but the full functions (4.17) are regular there. Note that $m$ runs now from 0 to $2 k-2$, which gives finally $k$ (rather than $k+1$ ) solutions in accordance with (1.7).

$\sigma$-model on the quotient. When counting the states, we first have found all the regular solutions of (4.15) for the functions having the form (4.14) (when staying at the tree level) or involving extra $z$-dependent factors as in (4.17) (when extra fluxes at the corners are taken into account). Then we imposed the Weyl invariance requirement.

Another way to handle this problem is to factorize our torus over the Weyl group and study the effective theory on the quotient [1]. For $\mathrm{SU}(2)$, the Weyl group is just $Z_{2}$. As is 
well known, $T^{2} / Z_{2}=S^{2}{ }^{15}$ Thus, the effective theory with all gauge constraints resolved represents a certain $\sigma$-model on $S^{2}$. What particular model is it?

At the level of $T^{2}$, the effective supercharges were evaluated to have the form (4.1). A mathematician would call this differential operator a twisted antiholomorphic derivative (twisting means adding an Abelian gauge field). The presence of extra Grassmann factor in $Q^{\text {eff }}$ promotes it to the twisted antiholomorphic exterior derivative. When going down onto the quotient, the supercharges should keep this form.

We are thus arriving at the twisted Dolbeault complex. The twist (e.g. the magnetic flux or the second Chern class of the gauge field) is a half of the twist on $T^{2}$. When extra fluxes due to fermion loops are taken into account, we obtain the twist $(2 k-2) / 2=k-1$. It is rather remarkable that this twisted Dolbeault complex is equivalent to the Dirac complex for the field of flux $k{ }^{16}$ The Dirac index on $S^{2}$ is equal to $k$.

Incorrect results could be obtained if

1. Not taking into account extra fluxes. This would give twist $k$ for the Dolbeault complex and twist $k+1$ for the Dirac complex. This is the number of states in pure CS theory.

2. Taking into account both fermion-induced and gluon-induced fluxes as in [2]. This would give $k+1$ for the Dolbeault twist and $k+2$ for the Dirac twist.

Also for other unitary groups, the index (1.6) coincides with the Dirac index on $\mathbb{C P}^{N-1}$ with a properly chosen gauge field. Adding gluon-induced fluxes would amount to the shift $k \rightarrow k+N$. If no extra fluxes were added, we would obtain the tree level result

$$
I(k, N)=[\operatorname{sgn}(k)]^{N-1}\left(\begin{array}{c}
|k|+N-1 \\
N-1
\end{array}\right) .
$$

which would make sense, for odd $N$, not for half-integer values of $k$, as it should [16], but for integer ones.

Our final remark is that, though the reduction of a complicated field theory problem to a much simpler problem of calculating the Dirac index on $\mathbb{C P}^{N-1}$ looks as a nice Christmas gift, we do not see any other way to prove that the corners contribute the flux that exactly compensates the flux associated with the square root of the canonical bundle (the difference between the Dirac twist and the Dolbeault twist) than to perform an accurate effective Hamiltonian analysis as we did in section 3 and section 4 above and in appendix $\mathrm{C}$ below.

\section{Acknowledgments}

I am indebted to E. Witten for many illuminating discussions and correspondence and to M. Konyushikhin for reading the manuscript and useful comments.

\footnotetext{
${ }^{15}$ One of the many ways to see it is meditating over figure 5 of ref. [2]. Note also that, for $\operatorname{SU}(N)$, the corresponding quotient is $\left[T^{\max } \times T^{\max }\right] / S_{N}=\mathbb{C P}^{N-1}[25]$.

${ }^{16} \mathrm{~A}$ mathematician can consult e.g. the Propositions 1.4.23 and 1.4.25 in the book [26] and a physicist may look into [27] for pedagogical explanations.
} 


\section{A Theta functions}

We remind here certain mathematical facts concerning the properties of analytical functions on the torus. They are mostly taken from the textbook [28], but we are using different notations which we find more clear and more appropriate for our purposes.

Theta functions play the same role for the torus as ordinary polynomials for the Riemann sphere. They are analytic, but satisfy certain nontrivial quasiperiodic boundary conditions with respect to shifts along the cycles of the torus. A generic torus is characterized by a complex modular parameter $\tau$, but we will stick to the simplest choice $\tau=i$ so that the torus represents a square $x, y \in[0,1](z=x+i y)$ glued around.

The simplest $\theta$-function satisfies the boundary conditions

$$
\begin{aligned}
\theta(z+1) & =\theta(z), \\
\theta(z+i) & =e^{\pi(1-2 i z)} \theta(z) .
\end{aligned}
$$

This defines a unique (up to a constant complex factor) analytic function. Its explicit form is

$$
\theta(z)=\sum_{n=-\infty}^{\infty} \exp \left\{-\pi n^{2}+2 \pi i n z\right\}
$$

This function (call it theta function of level 1 and introduce an alternative notation $\theta(z) \equiv$ $\left.Q^{1}(z)\right)$ has only one zero in the square $x, y \in[0,1]$ - right in its middle, $\theta\left(\frac{1+i}{2}\right)=0$.

For any integer $q>0$, one can define theta functions of level $q$ satisfying

$$
\begin{aligned}
& Q^{q}(z+1)=Q^{q}(z), \\
& Q^{q}(z+i)=e^{q \pi(1-2 i z)} Q^{q}(z) .
\end{aligned}
$$

When $q>1$, the functions satisfying (A.3) lie in vector space of dimension $q$. The basis in this vector space can be chosen as

$$
Q_{m}^{q}(z)=\sum_{n=-\infty}^{\infty} \exp \left\{-\pi q\left(n+\frac{m}{q}\right)^{2}+2 \pi i q z\left(n+\frac{m}{q}\right)\right\}, \quad m=0, \ldots, q-1 .
$$

$Q_{m}^{q}(z)$ can be expressed in the notation of [28] as

$$
Q_{m}^{q}(z)=\theta_{m / q, 0}(q z, i q),
$$

where $\theta_{a, b}(z, \tau)$ are theta functions of rational characteristics.

$Q_{m}^{q}(z)$ can be called "elliptic polynomials" of order $q$. Indeed, each $Q_{m}^{q}(z)$ has $q$ simple zeros at

$$
z_{s}^{(m)}=\frac{2 s+1}{2 q}+i\left(\frac{1}{2}-\frac{m}{q}\right), \quad s=0, \ldots, q-1
$$

(add $i$ to bring it onto fundamental domain $x, y \in[0,1]$ when necessary). A product $Q^{q}(z) Q^{q^{\prime}}(z)$ of two such "polynomials" of orders $q, q^{\prime}$ gives a polynomial of order $q+q^{\prime}$. 
For example, $\theta^{2}(z)$, having the zero of order 2, can be represented as a superposition

$$
\theta^{2}(z)=\alpha Q_{0}^{2}(z)+\beta Q_{1}^{2}(z) .
$$

The coefficients $\alpha, \beta$ can be determined. A great number of similar relations between theta functions of different levels can be written. We can amuse the reader with a relation

$$
\frac{Q_{5}^{6}-Q_{1}^{6}}{\left(Q_{3}^{4}-Q_{1}^{4}\right) Q_{0}^{2}}=\frac{1}{\eta(i)}=\frac{2 \pi^{3 / 4}}{\Gamma(1 / 4)}
$$

with some physical implications to be discussed soon.

A ratio of different theta functions of the same order

$$
R(z)=\frac{Q^{q}(z)}{\tilde{Q}^{q}(z)}
$$

represents a periodic meromorphic function. A properly defined number of zeros of this function (such that a zero of the second order is counted twice, etc) coincides with the properly defined number of its poles (the Riemann-Roch theorem).

\section{B Counting of states in pure CS theory}

We just outline here the main steps of the analysis of refs. [7, 8]. A reader is invited to look into the original papers and into the review [5] for more details.

The first remark is that the pure Chern-Simons is a topological theory involving zero Hamiltonian and a finite number of states. Their wave functions depend not on both $A_{1}$ and $A_{2}$ as is the case in the dynamical YM-CS theory, but rather on the antiholomorphic combination $\bar{A}=A_{-}=A_{1}-i A_{2}$, with $A_{+}=A_{1}+i A_{2}$ playing the role of canonical momenta. ${ }^{17}$

We put the theory on the spatial torus of size $L=1$ (as this theory does not involve dimensional constants, we cannot say whether the volume is large or small and will measure everything in the units of $L$ ).

A generic couple of matrix-valued Hermitian fields $A_{j}$ on the torus can be parametrized as $[31]$

$$
A_{1}-i A_{2}=2 \pi \mathrm{U}(\boldsymbol{\xi}) \bar{z} \sigma^{3} U^{-1}(\boldsymbol{\xi})-i \partial_{-} \mathrm{U}(\boldsymbol{\xi}) \cdot U^{-1}(\boldsymbol{\xi}),
$$

where $\xi_{1,2}$ are physical spatial coordinates, $\bar{z}=x-i y$ is a constant complex number. $U=\exp \left\{\left(i \alpha^{a}-\beta^{a}\right) \sigma^{a}\right\}$ is a $\mathrm{SL}\left(2, \mathbb{C}^{n}\right)$ matrix. When $\beta^{a}=0, U \in \mathrm{SU}(2)$ and (B.1) is reduced to a gauge transformed constant field. In this case (but not in a generic case), the conjugated field $A_{+}$can also be represented as in (B.1) with $\partial_{+}$being replaced for $\partial_{-}-$ see eq. (B.6) below.

\footnotetext{
${ }^{17}$ The reason by which the wave functions should be chosen to be antiholomorphic is explained in the paragraph after eq. (B.12). Note that, in refs. $[5,7,8]$ the wave functions depended on $A_{+}$rather than $A_{-}$ due to a different sign convention for $k$.
} 
The wave functions must satisfy the Gauss law constraints. In the pure CS case, they boil down to

$$
F_{12} \Psi[\bar{A}]=0
$$

The solution to these constraints is

$$
\Psi[\bar{A}]=\psi(\bar{z}) \exp \left\{-k S_{+}[U]-\frac{i k \bar{z}}{2} \int\left\langle\sigma^{3} U^{-1} \partial_{+} U\right\rangle d^{2} \xi\right\}
$$

with $S_{+}[U]$ being the Polyakov-Wiegmann functional [29],

$$
S_{+}[U]=\frac{1}{8 \pi} \int_{T^{2}}\left\langle U^{-1} \partial_{-} U U^{-1} \partial_{+} U\right\rangle+\frac{i}{12 \pi} \int_{(3)} \epsilon^{\mu \nu \rho}\left\langle U^{-1} \partial_{\mu} U U^{-1} \partial_{\nu} U U^{-1} \partial_{\rho} U\right\rangle .
$$

The integral in the second term runs over a 3-manifold with the border $T^{2}$ and $\langle\cdots\rangle$ stands for the trace.

To check the validity of (B.2), one should act on the wave function (B.3) by the operator

$$
A_{+}^{a}=\frac{2}{\kappa}\left(\frac{\delta}{\delta A_{1}^{a}}+i \frac{\delta}{\delta A_{2}^{a}}\right)=\frac{4}{\kappa} \frac{\delta}{\delta A_{-}^{a}}
$$

and be convinced (see $[5,7,8]$ for details) that one thus obtains a factor

$$
B_{+}^{a}=2 \pi U \bar{z} \sigma^{3} U^{-1}-i \partial_{+} U \cdot U^{-1}
$$

in front of $\Psi[\bar{A}]$, as if it were a pure gauge transformation. The differential operator $F_{12}=(i / 2) F_{+-}$gives then $\partial_{+} A_{-}-\partial_{-} B_{+}-i\left[B_{+}, A_{-}\right]$, which is zero.

On top of (B.2), one should require the wave functional (B.3) to be invariant with respect to two large (uncontractable) gauge transformations with the matrices

$$
U_{1}(\xi)=e^{2 \pi i \xi_{1} \sigma_{3}}, \quad U_{2}(\xi)=e^{-2 \pi i \xi_{2} \sigma_{3}}
$$

They correspond to the shifts $\bar{z} \rightarrow \bar{z}+1$ and $\bar{z} \rightarrow \bar{z}+i$. This brings $z$ onto the dual torus, $x, y \in[0,1]$. The invariance under (B.7) implies the boundary conditions

$$
\begin{aligned}
& \psi(\bar{z}+1)=e^{\pi k(1+2 \bar{z})} \psi(\bar{z}), \\
& \psi(\bar{z}+i)=e^{\pi k(1-2 i \bar{z})} \psi(\bar{z}) .
\end{aligned}
$$

And that means that

$$
\psi(\bar{z})=e^{\pi k \bar{z}^{2}} Q^{2 k}(\bar{z}),
$$

where $Q^{2 k}(z)$ is a theta function of level $2 k$.

Finally, we impose the requirement of Weyl invariance, $\psi(-\bar{z})=\psi(\bar{z})$. This reduces the number of states from $2 k$ (the dimension of the vector space of $Q^{2 k}(\bar{z})$ ) down to $k+1$. This gives

$$
\#_{\text {states }}(\text { pure } C S, \mathrm{SU}(2))=k+1
$$


Wave functions (B.3) can in principle be used to calculate certain averages, e.g. the Wilson loop averages related to knot invariants [6]. ${ }^{18}$ To this end, one should know the functional integral measure $\mathcal{D} A$. This measure was calculated in [30] with the result

$$
\left\langle\psi_{1} \mid \psi_{2}\right\rangle=\int d z d \bar{z} \psi_{1}^{*}(z) \psi_{2}(\bar{z}) e^{-2 \pi(k+2) z \bar{z}}|\Pi(z)|^{2},
$$

where $\Pi(z)$ is a certain theta function of level 4 having zeros at the "corners" of the dual torus, $\bar{z}=0,1 / 2, i / 2,(1+i) / 2$. In our notations,

$$
\Pi(z)=Q_{3}^{4}(z)-Q_{1}^{4}(z)
$$

It is antisymmetric in $z$.

Note that the measure involves the exponential factor $\exp \{-2 \pi(k+2) \bar{z} z\}$ which makes the integral convergent at large $|z|$. The negativity of the exponent there is due to the fact that our wave functions were chosen to be antiholomorphic. Holomorphic functions would lead to an inadmissible measure $\sim \exp \{2 \pi(k+2) \bar{z} z\}$.

For some purposes, it might be convenient to represent the Weyl invariant wave functions $Q^{2 k}(\bar{z})$ as a ratio of Weyl-antiinvariant functions of level $2(k+2)$ and the Weylantiinvariant function $\Pi(\bar{z})$, like in (A.8). Obviously, there are $(k+2)-1$ Weyl-antiinvariant functions $Q^{2(k+2)}(\bar{z})$, the number coinciding with (B.10). This works also for all other groups. The number of states can be counted as the number of generalized Weyl-invariant functions characterized by the integer $k$ or else as the number of Weyl-antiinvariant functions characterized by the integer $k+h$, where $h$ is the dual Coxeter number. However, if we are interested only in the state counting (as we are in this paper), and not in calculating averages, etc, the existence of the map $Q_{\text {Weyl inv. }}^{2 k} \rightarrow Q_{\text {Weyl antinv. }}^{2 k+4}$ is irrelevant.

This was all done for positive $k$. For negative $k$, wave functions depend on $A_{+}$rather than on $A_{-}$(such that the exponential factor in the measure provides, again, a suppression at large $|z|)$, but this is the only change. The whole analysis can be repeated with the result $|k|+1$ for the number of states.

\section{Wave function at the origin}

We will analyse here the ground states of the SQM Hamiltonian (3.8) and prove that, when $k>0$, the states with positive eigenvalues of the momentum (3.10) are necessarily singular at the origin and should be excluded from the spectrum. The case $k<0$ can be treated similarly, then negative $j$ are excluded.

A generic gauge-invariant bosonic wave function is written in eq. (3.11). The functions $P, S, R, T$ depend on three gauge-invariant variables $\mathcal{X}=A_{+}^{a} A_{-}^{a}, \mathcal{Z}=A_{+}^{a} A_{+}^{a}$ and $\overline{\mathcal{Z}}=$ $A_{-}^{a} A_{-}^{a}$. Consider the sector with a definite value of $j$. We can then write

$$
P=\left(\frac{\mathcal{Z}}{\overline{\mathcal{Z}}}\right)^{j / 4} \tilde{P}
$$

\footnotetext{
${ }^{18}$ We are not aware of such a direct calculation, however.
} 


$$
\begin{aligned}
S & =\left(\frac{\mathcal{Z}}{\overline{\mathcal{Z}}}\right)^{j / 4} \tilde{S}, \\
R & =\left(\frac{\mathcal{Z}}{\overline{\mathcal{Z}}}\right)^{(j-2) / 4} \tilde{R}, \\
T & =\left(\frac{\mathcal{Z}}{\overline{\mathcal{Z}}}\right)^{(j-1) / 4} \tilde{T},
\end{aligned}
$$

where $\tilde{R}, \tilde{S}, \tilde{R}$ and $\tilde{T}$ depend only on two neutral with respect to the charge (3.10) gaugeinvariant combinations $\mathcal{X}$ and $\mathcal{Y}=\mathcal{X}^{2}-\mathcal{Z} \overline{\mathcal{Z}}$. In the vicinity of the valley, they are reduced to $\mathcal{X} \rightarrow a^{2}$ and $\mathcal{Y} \rightarrow 4 a^{2} b^{2} \ll \mathcal{X}^{2}$.

Let us act now on the wave function (3.11) by the supercharges (3.3). We obtain a system of PDE of the first order for four functions $\tilde{P}, \tilde{S}, \tilde{R}, \tilde{T}$. One of these equations is actually algebraic, $\tilde{T}=0$. Three remaining functions satisfy three equations.

$$
\begin{aligned}
\mathcal{X} \tilde{S}+\sqrt{\mathcal{X}^{2}-\mathcal{Y}} \tilde{R} & =i \lambda(\partial \mathcal{X}+2 \mathcal{X} \partial \mathcal{Y}) \tilde{P}+i m \tilde{P} \\
\tilde{S}+\frac{\mathcal{X}}{\sqrt{\mathcal{X}^{2}-\mathcal{Y}}} \tilde{R} & =2 i \lambda\left[\partial \mathcal{Y}-\frac{j}{4\left(\mathcal{X}^{2}-\mathcal{Y}\right)}\right] \tilde{P} \\
\tilde{P} & =-i \lambda\left\{(\partial \mathcal{X}+2 \mathcal{X} \partial \mathcal{Y}) \tilde{S}+2 \sqrt{\mathcal{X}^{2}-\mathcal{Y}}\left[\partial \mathcal{Y}+\frac{j-2}{4\left(\mathcal{X}^{2}-\mathcal{Y}\right)}\right] \tilde{R}\right\}+i m \tilde{S}
\end{aligned}
$$

with $\lambda=4 g_{0}^{2}$. We are interested in the behavior of the solutions near the corner when $\mathcal{X} \ll \lambda / m$ [see (3.13)]. In this region, mass terms are not important and can be neglected. Let us do it and concentrate on the first two equations in (C.2) in the region $\mathcal{Y} \ll \mathcal{X}^{2}$ not necessarily assuming that $\mathcal{X} \gg g_{0}^{2 / 3}$ (the condition for the Abelian BO description to be valid). One can say that we are approaching the origin along the trace of the Abelian valley. Neglecting $\mathcal{Y}$ compared to $\mathcal{X}^{2}$, we readily see that the function $\tilde{P}(\mathcal{X}, 0)$ satisfies the equation

$$
\left(\frac{\partial}{\partial \mathcal{X}}+\frac{j}{2 \mathcal{X}}\right) \tilde{P}=0
$$

with the solution

$$
\tilde{P} \sim \mathcal{X}^{-j / 2}
$$

The behavior (C.4) coincides with (4.8) derived earlier. But it was derived there only in the region where the Abelian BO approximation is valid. The analysis of the full non-Abelian equations (C.2) allowed us here to extend this asymptotics down to the very origin.

\section{Supercharges, Hamiltonian and gauge-invariant variables}

The Hamiltonian analysis of section 3 can alternatively be done by resolving the gauge constraints at the classical level and expressing the supercharges and Hamiltonian into gauge-invariant variables. For the gauge SQM system obtained by reduction from $(3+1)$ 
SYM theory, this was done in [32]. We present here (mainly for methodical purposes) a similar analysis for the Hamiltonian (3.8).

Six dynamical variables $A_{j}{ }^{a}$ involve 3 gauge-invariant variables and 3 gauge angles. The latter can be effectively separated if using the polar representation [33, 34] ,

$$
A_{j}{ }^{a}=U_{j k} \Lambda_{k}^{b} V_{b a}
$$

where $U_{j k}(\alpha)$ is an $O(2)$ matrix describing spatial rotations, $V_{b a}\left(\phi^{a}\right)$ is an $O(3)$ gauge rotation matrix and $\Lambda_{k}^{b}$ is a quasidiagonal matrix,

$$
\Lambda_{k}^{b}=\left(\begin{array}{lll}
a & 0 & 0 \\
0 & b & 0
\end{array}\right) .
$$

By a proper spatial and/or gauge rotation the eigenvalues of $\Lambda_{k}^{b}$ can be brought to the range $a \geq|b|$. The fields (D.2) with positive or negative sign of $b$ are related to each other by a spatial reflection.

Gauge-invariant variables are thus $a, b, \alpha$, while $\phi^{a}$ are gauge angles. The quantum problem involves, generally speaking, two sectors: the even in $b$ and odd in $b$ wave functions. In the leading BO approximation, the wave functions are even (see eq. (D.11) below).

There are two ways to derive the expressions for gauge-invariant quantum supercharges and the Hamiltonian. First, one can resolve the constraints at the classical level and obtain classical gauge-invariant supercharges. For supersymmetry to be kept at the quantum level, one should resolve the ordering ambiguities in the supercharges using symmetric Weyl prescription. The quantum Hamiltonian is then obtained as the anticommutator $\{\bar{Q}, Q\} / 2 .{ }^{19}$ Such supercharges and the Hamiltonian act in the Hilbert space with "flat" measure $\sim d a d b d \alpha$. However, the 3-manifold of gauge-invariant variables $(a, b, \alpha)$ is in fact curved. If one is interested in the operators acting on the wave function normalized with the covariant measure

$$
\prod_{a j} d A_{j}^{a}=a|b|\left(a^{2}-b^{2}\right) d a d b d \alpha d \mu_{V} \longrightarrow C a|b|\left(a^{2}-b^{2}\right) d a d b d \alpha \equiv \mu_{a b} d a d b d \alpha,
$$

$\left(d \mu_{V}\right.$ is the Haar measure on $\left.\mathrm{SO}(3)\right)$, one should perform a proper similarity transformation and replace $Q^{\text {flat }}$ by

$$
Q^{\mathrm{cov}}=\frac{1}{\sqrt{\mu_{a b}}} Q^{\mathrm{flat}} \sqrt{\mu_{a b}}
$$

and, similarly, other operators.

Another approach is more direct and does not come to grips with a difficult ordering ambiguities problem,

1. We take the expressions (3.3), (3.8) for the quantum supercharges and Hamiltonian and express them into new variables.

\footnotetext{
${ }^{19}$ Note that this Hamiltonian does not coincide with the Weyl-ordered classical Hamiltonian (see ref. [32] for further details).
} 
2. Anticipating the eventual gauge fixing $\phi^{a}=0$, we only consider a simplified version of these expressions in the small $\phi^{a}$ region such that the body-frame gauge angular momenta $J^{a}=V^{a d} \epsilon^{d b c} A_{j}^{b} E_{j}^{c}$, in terms of which the Hamiltonian (3.8) is expressed, go over to the generators $J^{a} \rightarrow-i \partial / \partial \phi^{a}$.

3. The Gauss law constraints $\hat{G}^{a} \equiv 0$ allow one to express the latter via the fermion variables,

$$
J^{a} \equiv i \epsilon^{a b c} \psi^{b} \bar{\psi}^{c}
$$

This or other way, one obtains, assuming $b>0$,

$$
\begin{gathered}
Q^{\mathrm{cov}=}=e^{-i \alpha} g_{0}\left[\psi^{1}\left(p_{a}-i \frac{a p_{\alpha}+b J^{3}}{a^{2}-b^{2}}-\frac{i m a}{2 g_{0}^{2}}\right)+\psi^{2}\left(-i p_{b}+\frac{b p_{\alpha}+a J^{3}}{a^{2}-b^{2}}-\frac{m b}{2 g_{0}^{2}}\right)\right. \\
\left.-\psi^{3}\left(\frac{J^{2}}{a}+\frac{i J^{1}}{b}\right)\right]+\frac{i a b}{g_{0}} \bar{\psi}^{3} \\
\bar{Q}^{\mathrm{cov}}=g_{0} e^{i \alpha}\left[\bar{\psi}^{1}\left(p_{a}+i \frac{a p_{\alpha}+b J^{3}}{a^{2}-b^{2}}+\frac{i m a}{2 g_{0}^{2}}\right)+\bar{\psi}^{2}\left(i p_{b}+\frac{b p_{\alpha}+a J^{3}}{a^{2}-b^{2}}-\frac{m b}{2 g_{0}^{2}}\right)\right. \\
\left.-\bar{\psi}^{3}\left(\frac{J^{2}}{a}-\frac{i J^{1}}{b}\right)\right]-\frac{i a b}{g_{0}} \psi^{3}
\end{gathered}
$$

where $p_{a}=-i \partial / \partial a$, etc, and one should substitute for $J^{a}$ the fermion bilinears (D.5). The Hamiltonian is

$$
\begin{aligned}
H= & -\frac{g_{0}^{2}}{2} \triangle-\frac{i m}{2} \frac{\partial}{\partial \alpha}+\frac{1}{2 g_{0}^{2}}\left[a^{2} b^{2}+\frac{m^{2}}{4}\left(a^{2}+b^{2}\right)\right] \\
& +i \frac{\epsilon^{a b c}}{2}\left[\bar{\psi}^{a} \bar{\psi}^{b} A_{+}^{c}+\psi^{a} \psi^{b} A_{-}^{c}\right]+\frac{m}{2}\left(\psi^{a} \bar{\psi}^{a}-\bar{\psi}^{a} \psi^{a}\right),
\end{aligned}
$$

where

$$
A_{ \pm}^{1}=a e^{ \pm i \alpha}, \quad A_{ \pm}^{2}= \pm i b e^{ \pm i \alpha}, \quad A_{ \pm}^{3}=0 .
$$

and

$$
\begin{aligned}
\triangle= & \frac{\partial^{2}}{\left(\partial A_{i}^{a}\right)^{2}} \\
= & \frac{\partial^{2}}{(\partial a)^{2}}+\frac{\partial^{2}}{(\partial b)^{2}}+\frac{1}{a} \frac{\partial}{\partial a}+\frac{1}{b} \frac{\partial}{\partial b}+\frac{2}{a^{2}-b^{2}}\left(a \frac{\partial}{\partial a}-b \frac{\partial}{\partial b}\right) \\
& +\frac{1}{\left(a^{2}-b^{2}\right)^{2}}\left[\left(a^{2}+b^{2}\right)\left(\frac{\partial^{2}}{\partial \alpha^{2}}-\left(J^{3}\right)^{2}\right)+4 i a b J^{3} \frac{\partial}{\partial \alpha}\right]-\frac{\left(J^{2}\right)^{2}}{a^{2}}-\frac{\left(J^{1}\right)^{2}}{b^{2}}
\end{aligned}
$$

The Hamiltonian (D.7) is Hermitian with respect to the measure (D.3), $H^{\dagger}=\mu_{a b} H \mu_{a b}^{-1}$. The supercharges (D.6) satisfy $Q^{\dagger}=\mu_{a b} \bar{Q} \mu_{a b}^{-1}$. They are nilpotent and their anticommutator gives (D.7), as it should. These operators act on the wave functions normalized with the measure (D.3). The conserved angular momentum (3.10) is expressed as

$$
j=p_{\alpha}+\frac{1}{2} \psi^{a} \bar{\psi}^{a} .
$$


The expressions (D.6), (D.7) look complicated, but they are simplified a lot along the valleys. The slow bosonic variables are $a$ and $\alpha$. The combinations $a e^{ \pm i \alpha}$ coincide with the variables $C_{ \pm}$of section 3 . Now, $b^{2}$ is the fast variable, it corresponds to $\left(b_{j}^{a}\right)^{2}$ of section 3. The BO approximation works when $b_{\text {char }}^{2} \ll a^{2}$, which is true as long as $a \gg g_{0}^{2 / 3}$ as in (3.13). The fast massless Hamiltonian (3.15) is expressed as

$$
\begin{aligned}
H^{\text {fast }}= & -\frac{g_{0}^{2}}{2}\left[\frac{\partial^{2}}{(\partial b)^{2}}+\frac{1}{b} \frac{\partial}{\partial b}\right]+\frac{a^{2} b^{2}}{2 g_{0}^{2}}-\frac{g_{0}^{2}\left(\bar{\psi}^{2} \psi^{3}-\bar{\psi}^{3} \psi^{2}\right)^{2}}{2 b^{2}} \\
& +\frac{i a}{2} \epsilon^{a b}\left[e^{i \alpha} \bar{\psi}^{a} \bar{\psi}^{b}+e^{-i \alpha} \psi^{a} \psi^{b}\right],
\end{aligned}
$$

where $a, b=2,3$ and $\epsilon^{23}=1 .^{20}$

The fast ground state wave function (3.16) takes the form

$$
\Psi^{\text {fast }}\left(b, \psi^{2}, \psi^{3}\right) \sim \frac{1}{\sqrt{a}} \exp \left\{-\frac{a b^{2}}{2 g_{0}^{2}}\right\}\left(2 i+e^{-i \alpha} \epsilon^{a b} \psi^{a} \psi^{b}\right) .
$$

The analysis of section 4 remains intact. The analysis of appendix $\mathrm{C}$ can also be translated into new variables, $\mathcal{X}, \mathcal{Y} \rightarrow a, b$.

Open Access. This article is distributed under the terms of the Creative Commons Attribution License which permits any use, distribution and reproduction in any medium, provided the original author(s) and source are credited.

\section{References}

[1] E. Witten, Supersymmetric index of three-dimensional gauge theory, in The many faces of the superworld, M.A. Shifman and Y. Golfand ed., World Scientific, Singapore (1999), hep-th/9903005 [INSPIRE].

[2] A. Smilga, Witten index in supersymmetric 3D theories revisited, JHEP 01 (2010) 086 [arXiv:0910.0803] [INSPIRE].

[3] J. Bagger and N. Lambert, Gauge symmetry and supersymmetry of multiple M2-branes, Phys. Rev. D 77 (2008) 065008 [arXiv:0711.0955] [INSPIRE].

[4] O. Aharony, O. Bergman, D.L. Jafferis and J. Maldacena, $N=6$ superconformal Chern-Simons-matter theories, M2-branes and their gravity duals, JHEP 10 (2008) 091 [arXiv: 0806.1218] [INSPIRE].

[5] G.V. Dunne, Aspects of Chern-Simons theory, hep-th/9902115 [INSPIRE].

[6] E. Witten, Quantum field theory and the Jones polynomial, Commun. Math. Phys. 121 (1989) 351 [inSPIRE].

[7] S. Elitzur, G.W. Moore, A. Schwimmer and N. Seiberg, Remarks on the canonical quantization of the Chern-Simons-Witten theory, Nucl. Phys. B 326 (1989) 108 [InSPIRE].

[8] J. Labastida and A. Ramallo, Operator formalism for Chern-Simons theories, Phys. Lett. B 227 (1989) 92 [INSPIRE].

\footnotetext{
${ }^{20}$ Do not mix the color indices $a, b$ with the bosonic variables $a, b$ (sorry, but the Latin alphabet is not large...).
} 
[9] E. Witten, Constraints on supersymmetry breaking, Nucl. Phys. B 202 (1982) 253 [INSPIRE].

[10] B.A. Dubrovin, I.M. Krichever and S.P. Novikov, Schrödinger equation in a periodic field and Riemann surfaces, Dokl. Akad. Nauk SSSR 229 (1976) 15.

[11] B.A. Dubrovin and S.P. Novikov, Ground states of a two-dimensional electron in a periodic magnetic field, Zh. Eksp. Teor. Fiz. 79 (1980) 1006.

[12] A.V. Smilga, Noninteger flux: why it does not work, J. Math. Phys. 53 (2012) 042103 [arXiv: 1104.3986] [INSPIRE].

[13] S. Pancharatnam, Generalized theory of interference, and its applications, Proc. Indian Acad. Sci. A 44 (1956) 247.

[14] M. Berry, Quantal phase factors accompanying adiabatic changes, Proc. Roy. Soc. Lond. A 392 (1984) 45.

[15] R.D. Pisarski and S. Rao, Topologically massive chromodynamics in the perturbative regime, Phys. Rev. D 32 (1985) 2081 [inSPIRE].

[16] H.-C. Kao, K.-M. Lee and T. Lee, The Chern-Simons coefficient in supersymmetric Yang-Mills Chern-Simons theories, Phys. Lett. B 373 (1996) 94 [hep-th/9506170] [INSPIRE].

[17] A.V. Smilga, Vacuum structure in the chiral supersymmetric quantum electrodynamics, Sov. Phys. JETP 64 (1986) 8 [INSPIRE].

[18] B.Yu. Blok and A.V. Smilga, Effective zero mode hamiltonian in supersymmetric chiral nonabelian gauge theories, Nucl. Phys. B 287 (1987) 589 [INSPIRE].

[19] A.V. Smilga, Effective zero mode hamiltonian in supersymmetric chiral nonabelian gauge theories, Nucl. Phys. B 291 (1987) 241 [INSPIRE]

[20] A.V. Smilga, Born-Oppenheimer corrections to the effective zero mode Hamiltonian in SYM theory, JHEP 04 (2002) 054 [hep-th/0201048] [INSPIRE].

[21] M. Claudson and M.B. Halpern, Supersymmetric ground state wave functions, Nucl. Phys. B 250 (1985) 689 [INSPIRE].

[22] A.V. Smilga, Super-Yang-Mills quantum mechanics and supermembrane spectrum, in the proceedings of the International workshop on supermembranes and physics in $2+1$ dimensions, M.J. Duff, C.N. Pope and E. Sezgin eds., World Scientific, Singapore (1990).

[23] V. Kac and A.V. Smilga, Normalized vacuum states in $N=4$ supersymmetric Yang-Mills quantum mechanics with any gauge group, Nucl. Phys. B 571 (2000) 515 [hep-th/9908096] [INSPIRE].

[24] S. Deser, R. Jackiw and S. Templeton, Topologically massive gauge theories, Annals Phys. 140 (1982) 372 [Erratum ibid. 185 (1988) 406] [INSPIRE].

[25] R. Friedman, J. Morgan and E. Witten, Vector bundles and F-theory, Commun. Math. Phys. 187 (1997) 679 [hep-th/9701162] [INSPIRE].

[26] L.I. Nicolaescu, Notes on Seiberg-Witten theory, American Mathematical Society, Providence U.S.A. (2000).

[27] E. Ivanov and A. Smilga, Dirac operator on complex manifolds and supersymmetric quantum mechanics, arXiv: 1012.2069 [INSPIRE].

[28] D. Mumford, Tata lectures on theta, Birkhäuser, Boston U.S.A. (1983). 
[29] A.M. Polyakov and P. Wiegmann, Theory of nonabelian goldstone bosons, Phys. Lett. B 131 (1983) 121 [INSPIRE].

[30] K. Gawędzki and A. Kupiainen, Coset construction from functional integrals, Nucl. Phys. B 320 (1989) 625 [inSPIRE].

[31] C.N. Yang, Condition of selfduality for SU(2) gauge fields on euclidean four-dimensional space, Phys. Rev. Lett. 38 (1977) 1377 [INSPIRE].

[32] A.V. Smilga, How to quantize supersymmetric theories, Nucl. Phys. B 292 (1987) 363 [INSPIRE].

[33] H.M. Asatryan and G.K. Savvidy, Configuration manifold of Yang-Mills classical mechanics, Phys. Lett. A 99 (1983) 290 [INSPIRE].

[34] Y. Simonov, QCD hamiltonian in the polar representation, Yad. Fiz. 41 (1985) 1311 [INSPIRE]. 